\title{
EFEITO DO MANEJO DA IRRIGAÇÃO NA QUALIDADE DA PRODUÇÃO E NA PRODUTIVIDADE DO AMENDOIM CV. BR1
}

\author{
Luiz Carlos Silva ${ }^{1}$, Napoleão Esberard de Macêdo Beltrão ${ }^{2}$, \\ Tantravahi Venkata Ramana $\operatorname{Rao}^{3}$ e José Fideles Filho ${ }^{4}$
}

\begin{abstract}
RESUMO
Com o objetivo de se definir a lâmina e o intervalo de irrigação para a cultura do amendoim cv. BR1, um experimento foi conduzido em Rodelas, Bahia, nos anos de 1994 e 1995. O solo na área experimental foi classificado como Regossol com mais de $89 \%$ de areia no perfil de 0 a $80 \mathrm{~cm}$. A demanda evaporativa do ar foi superior a $8 \mathrm{~mm}$ por dia. Foram testados três lâminas totais de água, 300,500 e $700 \mathrm{~mm}$ e três intervalos de irrigação, 2, 4 e 6 dias, em delineamento de blocos ao acaso e esquema de análise fatorial $3 \times 3$, com irrigação por sulcos. Verificou-se que a produtividade de amendoim em grão e em casca foi significativamente afetada pelos dois fatores estudados, sendo o máximo, $3235 \mathrm{~kg} \cdot$ ha $^{-1}$ de amendoim em casca, obtido com lâmina total de água de $700 \mathrm{~mm}$ e intervalo de irrigação de 4 dias; o maior índice de colheita, de $37,4 \%$, foi obtido no tratamento com $700 \mathrm{~mm}$ de água e 6 dias de intervalo de irrigação, e o menor, $16,87 \%$, no tratamento com $300 \mathrm{~mm}$ de água e 4 dias de intervalo de irrigação, para a variável amendoim em grão.
\end{abstract}

Palavras-chave: Arachis hypogaea, índice de colheita, função de produção, teor de óleo

\section{EFFECT OF THE IRRIGATION MANAGEMENT ON PRODUCTION QUALITY AND PRODUCTIVITY OF THE GROUNDNUT CV. BR1}

\begin{abstract}
With the objective of defining the water depth and interval of irrigation of a groundnut crop, cultivar BR1, an experiment was conducted in Rodelas-BA during the years of 1994 and 1995. The soil at the experimental area was classified as Regossol, with more than $89 \%$ of sand in the depth profile of 0 to $80 \mathrm{~cm}$. The evaporative demand at the site was more than $8 \mathrm{~mm}$ per day. Furrow irrigation was used with a $3 \times 3$ factorial analysis scheme in a randomized block design with three different levels, 300; 500 and 700mm and three different intervals of irrigation, 2, 4 and 6 days. It was verified that the groundnut production, with and without shell, was significantly affected by both the factors under study. The maximum yield was obtained for the treatment of $700 \mathrm{~mm} / 4$ days and the minimum yield was obtained for the treatment of $300 \mathrm{~mm} / 6$ days. The yield index for the groundnut seeds was maximum, $37.48 \%$ for the treatment of $700 \mathrm{~mm} / 6$ days and the minimum, and $16.87 \%$ for the treatment of $300 \mathrm{~mm} / 4$ days.
\end{abstract}

Key words: Arachis hypogaea, yield index, production function, oil content

\footnotetext{
${ }^{1}$ Eng. Agrônomo Dr., Embrapa Algodão, CP 174, CEP 58107-720, Campina Grande, PB

${ }^{2}$ Eng. Agrônomo Dr., Embrapa Algodão, Campina Grande, PB

${ }^{3}$ Agrometeorologista, Ph.D., Prof., UFPB, DCA, CP 518, CEP 58109-970, Campina Grande, PB

${ }^{4}$ Meteorologista, Dr., EMEPA-PB, Estrada da Imbaúba, km 3, Lagoa Seca, PB
} 


\section{INTRODUÇÃO}

No desenvolvimento de projetos de irrigação e exploração racional das culturas e recursos hídricos, é necessário responder-se, basicamente, a duas perguntas: quanto e quando irrigar. A oferta de água para as plantas deve ser feita nas quantidades requeridas e na época certa, de modo a não comprometer seu rendimento nem a qualidade do produto, por deficiência ou excesso de água (Hewitt et al. 1980). Por outro lado, em situações em que a água é limitante, a utilização de irrigação com déficit permite maior retorno econômico que a de irrigação completa (English, 1990; Calheiros et al., 1996).

Em amendoim, a ocorrência de déficit hídrico nas fases de crescimento e desenvolvimento dos ginóforos e das vagens, acarreta decréscimo na produção, pela redução do número de vagens antes mesmo que pelo peso das vagens, e sementes (Boote et al., 1976); entretanto, alguns pesquisadores (Wright et al., 1991) afirmam que déficit hídrico durante o enchimento das vagens geralmente reduz o peso das sementes e das vagens.

Baseados na literatura disponível, Boote et al. (1982) concluíram que cerca de $6000 \mathrm{~m}^{3}$.ha ${ }^{-1}$ de água são requeridos para o desempenho ótimo da produção de amendoim em casca. Desai et al. (1984) comparando as produções de amendoim em resposta a várias lâminas de irrigação, verificaram que tanto o excesso quanto a deficiência de água reduzem a produtividade do amendoim.

Nageswara Rao et al. (1988) aplicando lâminas de água de $725 \mathrm{~mm}, 630 \mathrm{~mm}, 580 \mathrm{~mm}$ e $550 \mathrm{~mm}$, obtiveram produtividades de amendoim em casca de 4615kg.ha- ${ }^{-1}, 5480 \mathrm{~kg} \cdot \mathrm{ha}^{-1}, 5040 \mathrm{~kg} \cdot \mathrm{ha}^{-1} \mathrm{e}$ $3687 \mathrm{~kg} \cdot \mathrm{ha}^{-1}$, respectivamente, evidenciando que água, em demasia quanto em deficiência, reflete negativamente na produção. Também, Távora \& Melo (1991) verificaram que a deficiência hídrica determinou redução média na produção de vagens de amendoim, da ordem de $62 \%$ em relação ao tratamento sem deficiência hídrica.

Ferreira et al. (1992) constataram reduções de até 75,5\% na produtividade de amendoim em casca, sob condições de severo estresse hídrico, quando comparado ao tratamento sem estresse.

O déficit hídrico reduz frequentemente o peso das sementes (Pallas et al., 1979) e porcentagem de sementes "extra grandes" (Stansell et al., 1976) enquanto aumenta a porcentagem de sementes danificadas ou enrugadas (Boote et al., 1982). A irrigação não tem efeitos significativos nem consistentes sobre a concentração de óleo e proteínas das sementes (Boote et al., 1982).

O objetivo deste trabalho foi verificar os efeitos do manejo da irrigação na qualidade da produção e na produtividade do amendoim cv. BR1, nas condições da borda do Lago de Itaparica, município de Rodelas, BA.

\section{MATERIAL E MÉTODOS}

O experimento foi conduzido nos anos de 1994 e 1995, na Estação Experimental da Companhia de Desenvolvimento do Vale do São Francisco (CODEVASF) situada no município de Rodelas, BA, cujas coordenadas geográficas são 0850'S de latitude, $38^{\circ} 46^{\prime} \mathrm{W}$ de longitude e altitude de $270 \mathrm{~m}$.

De acordo com a classificação de Köppen, o clima local é do tipo BSwh, correspondente a um clima muito quente, semiárido e com estação chuvosa limitada aos meses de janeiro a abril, sendo que as precipitações apresentam alta variabilidade espacial e temporal. Nos meses de agosto a dezembro, correspondentes ao período de execução do trabalho, não houve precipitação pluvial e, se observou que a temperatura máxima variou de $29,8^{\circ} \mathrm{C}$ a $35,9^{\circ} \mathrm{C}$, a mínima de $19,7^{\circ} \mathrm{C}$ a $23,6^{\circ} \mathrm{C}$, a média de $24,9^{\circ} \mathrm{C}$ a $29,1^{\circ} \mathrm{C}$, a umidade relativa do ar de 43 a $57 \%$, a evaporação do Tanque Classe A de $7,3 \mathrm{~mm}$ a $11,3 \mathrm{~mm}$ e a velocidade do vento de $5,85 \mathrm{~km} \cdot \mathrm{h}^{-1}$ a $10,5 \mathrm{~km} \cdot \mathrm{h}^{-1}$.

O solo foi classificado como Regossol de textura arenosa, cujas características físico-químicas e hídricas são apresentadas nas Tabelas 1 e 2; os dados foram determinados no Laboratório de Irrigação e Salinidade do Departamento de Engenharia Agrícola da Universidade Federal da Paraíba, UFPB.

Tabela 1. Características químicas do solo da área experimental a profundidade de 0 a $20 \mathrm{~cm}$. (CODEVASF) Rodelas, BA

Complexo sortivo (meq/100g de solo)

\begin{tabular}{cccccccccc}
\hline $\mathrm{Ca}$ & $\mathrm{Mg}$ & $\mathrm{Na}$ & $\mathrm{K}$ & $\mathrm{S}$ & $\mathrm{Al}$ & $\mathrm{pH}$ & $\mathrm{P}(\mathrm{ppm})$ & $\mathrm{N}(\%)$ & M.O.(\%) \\
\hline 1,78 & 0,65 & 0,06 & 0,003 & 2,5 & 0,1 & 6,48 & 3,74 & 0,03 & 0,55 \\
\hline
\end{tabular}

Tabela 2. Características físico-hídricas (capacidade de campo - CC, ponto de murcha - PM, densidade aparente - Da) e granulometria do solo da área experimental. (CODEVASF) Rodelas, BA

\begin{tabular}{ccccccc}
\hline $\begin{array}{c}\text { Profundidade } \\
(\mathrm{cm})\end{array}$ & $\begin{array}{c}\text { CC } \\
(\%)^{*}\end{array}$ & $\begin{array}{c}\text { PM } \\
(\%)^{*}\end{array}$ & $\begin{array}{c}\text { Da } \\
(\%)\end{array}$ & $\begin{array}{c}\text { Areia } \\
(\%)\end{array}$ & $\begin{array}{c}\text { Silte } \\
(\%)\end{array}$ & $\begin{array}{c}\text { Argila } \\
0-20\end{array}$ \\
\hline, 39 & 1,16 & 1,45 & 88,00 & 4,69 & 7,31 \\
$20-40$ & 3,88 & 1,34 & 1,49 & 89,01 & 2,67 & 8,32 \\
$40-60$ & 3,90 & 1,31 & 1,50 & 89,07 & 1,63 & 9,29 \\
$60-80$ & 4,61 & 1,31 & 1,49 & 89,07 & 1,64 & 9,29 \\
\hline
\end{tabular}

*(\%) com base na massa seca

O preparo do solo, dadas as condições de estrutura e textura, foi feito por duas gradagens cruzadas, usando-se uma grade leve de discos e a cultivar utilizada foi a BR1, sugerida para plantio pela Embrapa Algodão (CNPA).

O plantio foi realizado nos dias 06/08/94 e 14/09/95, para os $1^{\circ}$ e $2^{\circ}$ anos, respectivamente, usando-se 12 a 15 sementes por metro linear, semeadas a uma profundidade de 3 a $5 \mathrm{~cm}$, em sulcos com espaçamento de $0,50 \mathrm{~m}$.

Realizou-se o desbaste aos 25 dias após a semeadura, deixando-se 10 plantas/m e se obtendo uma densidade populacional de aproximadamente 200.000 plantas por hectare, enquanto as ervas daninhas foram controladas com o uso de herbicidas aplicados em pré-emergência, associados ao cultivo mecânico com o uso de enxada na fase mais avançada da cultura, sempre que houve necessidade.

O controle das pragas e das doenças foi realizado de acordo com as recomendações de Silva et al. (1993) e a colheita, manual, realizou-se aos 98 e 91 dias após a semeadura (DAS) nos anos de 1994 e 1995, respectivamente.

Todas as parcelas foram uniformemente irrigadas até o vigésimo quinto dia após a semeadura (DAS) quando foram implantados os tratamentos. A partir desta data, a irrigação foi realizada de maneira diferenciada e o sistema adotado foi o de sulcos em nível, fechados no final, com adução de água por tubos janelados, espaçados de $0,50 \mathrm{~m}$. e cada sulco media $6 \mathrm{~m}$ de comprimento. Para um controle mais eficiente das lâminas de água aplicadas, fez-se uso de um hidrômetro, com precisão de um litro.

Após a colheita, foram avaliados a influência das lâminas e os intervalos de irrigação sobre a produção de amendoim em casca, produção de grãos, rendimento relativo (sementes/vagens), peso de 100 sementes, peso de 100 vagens, percentagem de sementes perfeitas, percentagem de vagens chochas e teor de óleo (\%). Foram feitas, também, determinações do índice de colheita, funções de produção e superfícies de resposta.

$\mathrm{O}$ delineamento experimental foi o de blocos ao acaso, com esquema de análise fatorial $3 \times 3$, sendo os fatores três lâminas 
de água e três intervalos de irrigação, com 4 repetições. Cada unidade experimental foi constituída de 10 fileiras de plantas espaçadas de $0,5 \mathrm{~m}$, com $6 \mathrm{~m}$ de comprimento e área total de $30 \mathrm{~m}^{2}$, tendo como parcela útil as duas fileiras centrais, com área de $6 \mathrm{~m}^{2}$. Foram realizadas análises de variância, de correlação simples e de regressão, através de polinômios ortogonais e regressão não linear nos parâmetros do modelo, usando-se o método iterativo de Marquardt (1963). Foi, também, realizada análise conjunta para as variáveis estudadas, usando-se o modelo de split-plot, tendo como parcela principal os fatores lâminas e intervalos de irrigação e, como subparcela, os anos 1994 e 1995. $\mathrm{Na}$ determinação das funções de produção e superfícies de respostas foi usado o software S.A.S (Statistical Analsis System) versão 6, de 1994.

Os tratamentos adotados foram lâminas totais de irrigação de $700 \mathrm{~mm}, 500 \mathrm{~mm}$ e $300 \mathrm{~mm}$, e intervalos de irrigação de 2,4 e 6 dias.

\section{RESULTADOS E DISCUSSÃO}

Após a análise, verificou-se que, para todas as variáveis estudadas, os resultados foram estatisticamente iguais nos dois anos de condução do experimento, e em consequiência disto, serão apresentados e discutidos apenas os resultados da análise conjunta.

A percentagem de vagens chochas, embora com valores baixos, foi influenciada tanto pela lâmina quanto pelo intervalo de irrigação, variando de 3,5 a $8,5 \%$ na lâmina de $300 \mathrm{~mm}$, de 3,75 a $4,75 \%$ na lâmina de $500 \mathrm{~mm}$ e de 1,75 a $4,75 \%$ na lâmina de $700 \mathrm{~mm}$. Esses valores são indicadores de que o suprimento de cálcio mais magnésio na zona de desenvolvimento das vagens era satisfatório, visto que a deficiência de cálcio para a planta do amendoim é o principal fator responsável pela formação de vagens chochas, conforme atestam diversos autores citados por Silva et al. (1993).

A percentagem de sementes perfeitas variou de 95,5 a $100 \%$ e não houve diferença significativa a nível de $5 \%$ de probabilidade, pelo teste F. Esses resultados confirmam as observações efetuadas para a percentagem de vagem chocha.

$O$ peso de 100 sementes foi influenciado pelo intervalo de irrigação, mas não se verificou efeito significativo em relação às lâminas aplicadas, variando de 44,25g no intervalo de 2 dias, a 48,50g no intervalo de 6 dias, o que está de acordo com as características agronômicas e tecnológicas da cultivar. Quanto ao peso de 100 vagens, observaram-se as mesmas tendências do peso de 100 sementes; esses resultados estão de acordo com os resultados de Wright et al., (199)1, que afirmam haver, freqüentemente, redução no peso das sementes do amendoim, quando submetido a déficit hídrico.

O teor de óleo não apresentou diferença significativa entre as lâminas, mas foi altamente significativo o efeito de intervalos de irrigação, que variou de 46,66\% no intervalo de 2 dias, a $48,39 \%$ no intervalo de 6 dias, estando acima dos padrões tecnológicos indicados para a cultivar, que é, em média, de $45 \%$. Para muitos autores, como Boote et al., 1982, entre outros, a irrigação não tem efeitos significativos nem consistentes sobre a concentração de óleo nas sementes.

O rendimento relativo em sementes (\%) ou relação grãos/vagens, que para a cultivar é em média de $72 \%$, variou de $58,25 \%$ no tratamento de $300 \mathrm{~mm}$ com intervalo de 6 dias, entre irrigações a $72,25 \%$ no tratamento de $700 \mathrm{~mm}$ com intervalo de 4 dias entre as irrigações, sendo altamente significativos os efeitos de lâminas e intervalos de irrigação. A baixa relação grãos/vagens, associada a outros fatores, como menor peso das sementes e número de vagens por planta, contribuiu para a redução da produtividade de grãos de amendoim nos tratamentos com deficiência hídrica

Quando se analisa o efeito das lâminas de água, observa-se que a menor lâmina aplicada, 300mm, proporcionou o menor rendimento de amendoim em casca e em grão, o que indica que esta quantidade de água não foi suficiente para suprir as necessidades da cultura Tabela 3, Doorenbos \& Kassam (1979) afirmam que o consumo hídrico do amendoim situa-se entre 500 e 700mm, enquanto Nageswara Rao et al. (1988) confirmam esses resultados, visto que obtiveram menores produtividades de amendoim em casca com a aplicação de menores lâminas de irrigação, evidenciando que água em deficiência reflete negativamente na produtividade, fato confirmado por Ferreira et al. (1992) e Metochis (1993) que observaram queda no rendimento da cultura do amendoim, provocada pela deficiência de umidade.

Tabela 3. Produtividade e índice de colheita de amendoim, em casca e grão, submetido a diferentes lâminas e intervalos de irrigação, Rodelas, BA, 1994/95

\begin{tabular}{cccccc}
\hline & \multicolumn{2}{c}{ Produtividade $\left(\mathrm{kg} . \mathrm{ha}^{-1}\right)$} & & \multicolumn{2}{c}{ Índice de colheita $(\%)$} \\
\cline { 2 - 3 } \cline { 6 - 6 } Tratamento & Casca & Grão & & Casca & Grão \\
\hline $700 \mathrm{~mm} / 2$ dias & 2166 & 1644 & & 35,18 & 26,70 \\
$700 \mathrm{~mm} / 4$ dias & 3235 & 2026 & & 48,13 & 30,14 \\
$700 \mathrm{~mm} / 6$ dias & 2267 & 1554 & & 54,67 & 37,48 \\
$500 \mathrm{~mm} / 2$ dias & 2475 & 1671 & & 43,56 & 29,41 \\
$500 \mathrm{~mm} / 4$ dias & 1975 & 1384 & & 48,60 & 34,06 \\
$500 \mathrm{~mm} / 6$ dias & 1790 & 1093 & & 47,64 & 29,09 \\
$300 \mathrm{~mm} / 2$ dias & 1791 & 1232 & & 40,97 & 28,18 \\
$300 \mathrm{~mm} / 4$ dias & 1309 & 859 & 25,72 & 16,87 \\
$300 \mathrm{~mm} / 6$ dias & 970 & 609 & 22,25 & 17,15 \\
\hline
\end{tabular}

Analisando-se o efeito do intervalo de irrigação, observou-se que o menor rendimento ocorreu no tratamento cujo intervalo de irrigação foi de seis dias. Este resultado pode estar relacionado com as características do solo, uma vez que a alta percentagem de areia e a baixa percentagem no teor de matéria orgânica resultaram em pequena capacidade de retenção de água, ou seja, $1,31 \%$ de água disponível.

Com relação à partição de assimilados, configurada no coeficiente de migração ou índice de colheita, pode-se observar, na Tabela 3, que a relação em porcentos ponderais entre drenos úteis (frutos) e não úteis (vegetal restante) do ponto de vista de comercialização variou consideravelmente entre os tratamentos, denotando-se os decréscimos verificados quando da aplicação da lâmina de 300mm com intervalos de irrigação de 4 e 6 dias, comparados ao tratamento de $700 \mathrm{~mm}$, com 6 dias, que foi o que promoveu maior índice de colheita efetivo (grãos) de $37,48 \%$. De fato, com menor disponibilidade de água, as plantas desenvolveram mais fitomassa vegetativa, visando à sobrevivência, como foi evidenciado nos valores obtidos com a menor lâmina e intervalos mais espaçados, 4 e 6 dias.

Efetuada a análise de variância, obteve-se significância para a interação lâmina vs. intervalo de irrigação. Feitos os desdobramentos, verificou-se significância tanto para a lâmina quanto para o intervalo de irrigação e, da análise de regressão, obtiveram-se as funções de produção e superfícies de respostas, apresentadas nas Figuras 1 e 2. 


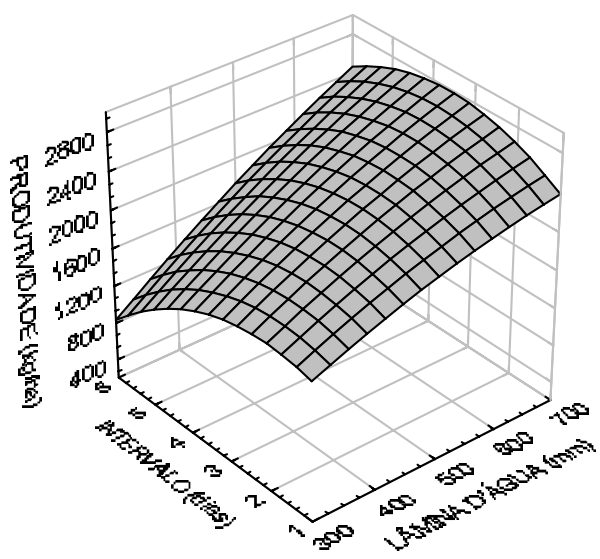

Figura 1. Superfície de respo sta para produtividade de amendoim em casca, em função da lâmina e do intervalo de irrigação

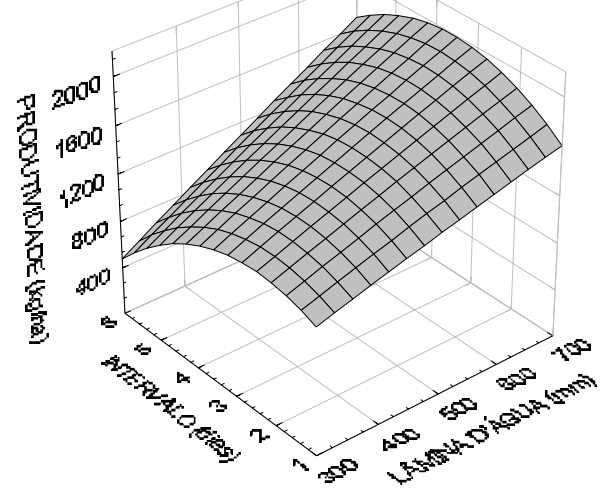

Figura 2. Superfície de resposta para produtividade de grão de amendoim em função da lâmina e do intervalo de irrigação

As funções de produção obtidas foram:

$\mathrm{Y}_{\mathrm{g}}=487,63+1,334 . \mathrm{L}+168,28 . \mathrm{I}-0,00032 . \mathrm{L}^{2}-55,31 . \mathrm{I}^{2}+0,3333 . \mathrm{L} . \mathrm{I}$ $Y_{c}^{g}=603,08+3,625 . L+100,37 . I-0,0023 . L^{2}-57,6 . I^{2}+0,454 . L . I$

em que:

$\mathrm{Y}_{\mathrm{g}}$ - o rendimento esperado de grãos de amendoim $\left(\mathrm{kg} \cdot \mathrm{ha}^{-1}\right)$ $\mathrm{Y}_{\mathrm{c}}^{\mathrm{g}}$ - o rendimento esperado de amendoim em casca $\left(\mathrm{kg}^{\mathrm{h}} \mathrm{ha}^{-1}\right)$

$\mathrm{L}^{\mathrm{c}}$ - a lâmina de água a ser aplicada em $\mathrm{mm}$ e

I - o intervalo de irrigação, em dias.

A solução dessas funções dentro dos seus domínios, permite estimar que a aplicação de $700 \mathrm{~mm}$ de água e intervalo de irrigação de 4 dias, possibilita a produtividade de $2765 \mathrm{~kg} \cdot \mathrm{ha}^{-1}$ de amendoim em casca ou 1986kg.ha-1 de grão.

\section{CONCLUSÕES}

1. A produtividade de grãos de amendoim e de amendoim em casca, foi afetada pela diferenciação das lâminas e pelos intervalos de irrigação, variando de $609 \mathrm{~kg} \cdot \mathrm{ha}^{-1}$ no tratamento de $300 \mathrm{~mm}$ de água e intervalo de irrigação de 6 dias, a 2026.ha ${ }^{-1}$ no tratamento de $700 \mathrm{~mm}$ de água e intervalo de irrigação de 4 dias, para grãos de amendoim e de $970 \mathrm{~kg} \cdot \mathrm{ha}^{-1}$ a $3235 \mathrm{~kg} \cdot \mathrm{ha}^{-1}$ de amendoim em casca, respectivamente.

2. A função de produção determinada para grãos foi do tipo quadrática, onde a aplicação de 700mm de água e intervalos de 4 dias possibilitaram a produtividade de $2765 \mathrm{~kg}^{-\mathrm{ha}^{-1}} \mathrm{de}$ amendoim em casca, ou $1986 \mathrm{~kg}$.ha-1 de grãos.

3. O coeficiente de migração do amendoim, tanto para o produto em casca como em grão, foi afetado pela lâmina aplicada e pelo intervalo de irrigação, com diferenças superiores a $100 \%$.

\section{REFERÊNCIAS BIBLIOGRÁFICAS}

BOOTE, K.J.; STANSELL, J.R.; SCHUBERT, A.M.; STONE, F.F. Irrigation, water use and water relations. In: Peanut Science and Technology. Yoakum, Texas: American Peanut Research and Education Society, 1982. p.164-205.

BOOTE, K.J.; VARNELL, R.J.; DUNCAN, W.G. Relationships of size, osmotic concentration, and sugar concentration of peanut pods to soil water. Proceedings Soil and Crop Science Society of Florida, v.35, p.47-50, 1976.

CALHEIROS, C.B.M.; QUEIROZ, J.E.; FRIZZONE, J.A.; PESSOA, P.C.S. Estratégias ótimas de irrigação do feijoeiro: água como fator limitante da produção. Pesquisa Agropecuária Brasileira, Brasília, v.31, n.7, p.509-515, 1996.

DESAI, N.D.; JOSHI, R.S.; PATEL, K.R. Response of summer groundnut to varius levels of irrigation on clay soils. Madras Agricultural Journal. v.9, p.617-620, 1984.

DOORENBOS, J.; KASSAM, A.H. Efectos del agua sobre el redimiento de los cultivos. Roma: FAO, 1979. 212 p. (Riego e Drenaje, 33)

ENGLISH, M.J. Deficit irrigation. 1. Analitical framework. Journal of Irrigation and Drainage Engineering,_New York, v.116, n.3, p.339-412, 1990.

FERREIRA, L.G.R.; SANTOS, I.F. dos; TÁVORA, F.J.F.; SILVA, J.V. da. Déficit hídrico em cultivares de amendoim (Arachis hypogaea L.). Respostas fisiológicas e produção. Oléagineux, v.47, n.8-9, p.523-530, 1992.

HEWITT, T.D.; GORBET, D.W.; WESTBERRY, G.O. Economics of irrigating peanuts. Proceedings Soil and Crop Science of Florida, v.39, p.135-140, 1980.

MARQUARDT, D. An algorithm for least squares estimation of non-linear parameters. SIAM Journal of Applied Mathematics, v.11, p.431-441, 1963.

METOCHIS, C. Irrigation of groundnut (Arachis hypogaea L.) grown in a Mediterranean environment. Cambridge. Journal of Agricultural Science, v.121, p.343-6, 1993.

NAGESWARA RAO, R.C.; WILLIAMS, J.H.; SIVAKUMAR, M.V.K.; WADIA, K.D.R. Effect of water deficit at different growth phases of peanut. 1l. Response to drought during preflowering phase. Agronomy Journal, v.80, p.431-438, 1988.

PALLAS JUNIOR, J.E.; STANSELL, J.R.; KOSKE, T.J. Effects of drought on florunner peanuts. Agronomy Journal, v.71, n.5, p.853-858, 1979.

SAS INSTITUTE. SAS/STAT user's guide; version 6. 4. ed. Cary, 1994. 1686p. v.1-2

SILVA, L.C.; MOREIRA, J. de A.N.; TAVARES SOBRINHO, J.; BELTRÃO, N.E. de M. Recomendações técnicas para o cultivo do amendoim no Nordeste Brasileiro. Campina Grande: EMBRAPA-CNPA, 1993. 26p. (EMBRAPA-CNPA. Circular Técnica, 16).

STANSELL, J.R.; SHEPHERD, J.L.; PALLAS J.E.; BRUCE, R.R.; MILTIN, N.A.; BELL, D.K.; MORGAN, W. Peanut responses to soil water variables in the Southeast. Peanut Science, v.3, p.44-48, 1976.

TÁVORA, F.J.A.F; MELO, F.I.O. Resposta de cultivares de amendoim a ciclos de deficiência hídrica: crescimento vegetativo, reprodutivo e relações hídricas. Ciência Agronômica, Fortaleza, v.22, n.1/2, p.47-60, 1991.

WRIGHT, G.C.; HUBICK, K.T.; FARQUHAR, G.D. Physiological analysis of peanut cultivar response to timing and duration of drought stress. Australian Journal for Agricultural Research, v.42, p.453-470, 1991. 